\title{
Cosmological singularities and modified theories of gravity
}

\author{
Leonardo Fernández-Jambrina* and Ruth Lazkoz \\ ${ }^{*}$ Matemática Aplicada, E.T.S.I. Navales, Universidad Politécnica de Madrid, \\ Arco de la Victoria s/n, E-28040 Madrid, Spain \\ ${ }^{\dagger}$ Física Teórica, Facultad de Ciencia y Tecnología, Universidad del País Vasco, \\ Apdo. 644, E-48080 Bilbao, Spain
}

\begin{abstract}
We consider perturbative modifications of the Friedmann equations in terms of density corresponding to modified theories of gravity proposed as an alternative route to comply with the observed accelerated expansion of the universe. Assuming that the present matter content of the universe is a pressureless fluid, the possible singularities that may arise as the final state of the universe are surveyed. It is shown that, at most, two coefficients of the perturbative expansion of the Friedman equations are relevant for the analysis. Some examples of application of the perturbative scheme are included.
\end{abstract}

Keywords: Dark energy, modified gravity, singularities, Friedmann equation

PACS: 04.20.Dw, 98.80.Jk, 95.36.+x, 04.50.+h

\section{INTRODUCTION}

Recent astronomical data from Type Ia supernovae [1] as well as from the CMB spectrum [2] confirm that our universe is undergoing an accelerated expansion period. In order to comply with this feature, one may resort to postulating a dark energy content for the universe [3], with undesired properties, such as violation of some energy conditions, or going beyond general relativity in the quest for another theory of gravity $[4,5]$.

With either approaches for dealing with the observed accelerated expansion, cosmology is much richer than it was thought in the previous century. According to classical cosmologies, the universe started at an initial singularity, the Big Bang, and it was doomed to expand forever, since the matter content was not dense enough to stop expansion and collapse into a final singularity.

\section{MODIFIED GRAVITY}

Instead of dealing with a full theory of modified gravity, we focus on the consequences at the level of cosmological equations, namely Friedmann equation, just requiring that it admits a generalised power expansion on the density $\rho$ around a value $\rho_{*}$,

$$
\left(\frac{\dot{a}}{a}\right)^{2}=H^{2}=h_{0}\left(\rho-\rho_{*}\right)^{\xi_{0}}+h_{1}\left(\rho-\rho_{*}\right)^{\xi_{1}}+\cdots, \quad \xi_{0}<\xi_{1}<\cdots .
$$


TABLE 1. Singularities in FLRW cosmological models

\begin{tabular}{ccccc}
\hline$\eta_{0}$ & $\eta_{1}$ & $\eta_{2}$ & Tipler & Królak \\
\hline$(-\infty, 0)$ & $\left(\eta_{0}, \infty\right)$ & $\left(\eta_{1}, \infty\right)$ & Strong & Strong \\
0 & $(0,1)$ & $\left(\eta_{1}, \infty\right)$ & Weak & Strong \\
& 1 & $(1,2)$ & Weak & Weak \\
& & {$[2, \infty)$} & Weak & Weak \\
& $(1,2)$ & $\left(\eta_{1}, \infty\right)$ & Weak & Weak \\
& {$[2, \infty)$} & $\left(\eta_{1}, \infty\right)$ & Weak & Weak \\
$(0, \infty)$ & $\left(\eta_{0}, \infty\right)$ & $\left(\eta_{1}, \infty\right)$ & Strong & Strong \\
\hline
\end{tabular}

The standard density term arises as the lineal term with an exponent equal to one and the cosmological constant appears with null exponent in this expansion. Further terms are interpreted as modifications of the theory.

On the other hand, the energy conservation law implies

$$
\dot{\rho}+3 H(\rho+p)=0,
$$

but assuming that the accelerated expansion of the universe is solely due to the modification of the theory of gravity, we choose a pressureless dust as matter content of the universe, so that the scale factor of the universe and the density are related by $\rho a^{3}=K$.

Thereby we may get rid of the scale factor and write down a modified Friedmann equation in terms of density,

$$
\frac{\dot{\rho}}{\rho}=-3 \sqrt{h_{0}}\left(\rho-\rho_{*}\right)^{\xi_{0} / 2}-\frac{3}{2} \frac{h_{1}}{\sqrt{h_{0}}}\left(\rho-\rho_{*}\right)^{\xi_{1}-\xi_{0} / 2}+\cdots .
$$

Solving this equation provides a perturbative expansion of the density in coordinate time, which we want to compare with a similar expansion for the scale factor,

$$
a(t)=c_{0}\left|t-t_{0}\right|^{\eta_{0}}+c_{1}\left|t-t_{1}\right|^{\eta_{1}}+\cdots, \quad \eta_{0}<\eta_{1}<\cdots,
$$

in terms of coordinate time. This is useful, since in [6] we have related the exponents $\eta_{i}$ with the strength of singularities [7, 8], as we see in Table 1.

Inserting the modified Friedmann equation into the conservation equation one gets:

Our purpose it to integrate the latter by considering all the possibilities which arise from different values of the parameters, and then use the aforementioned map between the energy density and the scale factor so that we can finally obtain asymptotic expressions for the expansionary behaviour of the models. Then, we will identify the specific late-time behaviour of the models, focusing on the existence of future singularities of various types. This classification resorts to earlier works by ourselves. 


\section{$<$ A subsubsection>}

Et iam nox umida caelo praecipitat $J_{\text {ion }}$ suadentque cadentia sidera somnos. Sed si tantus amor casus cognoscere nostros et breviter Troiae $J_{i o n}$ supremum audire laborem:

$$
J_{\text {ion }}=A \frac{\exp \left[-\frac{E_{a}}{k T}\right]}{k T} \alpha
$$

lamentabile regnum cruerint Danai; quaeque ipse miserrima vidi, et quorum pars magna fui. $A$ talia fando, $E_{a}$ iam nox umida, $k$ caelo praecipitat, suadentque cadentia sidera somnos. See (4).

\section{ACKNOWLEDGMENTS}

L.F.-J. is supported by the Spanish Ministry of Education and Science Project FIS-200505198. R.L. is supported by the University of the Basque Country through research grant GIU06/37 and by the Spanish Ministry of Education and Culture through research grant FIS2007-61800.

\section{REFERENCES}

1. T. M. Davis et al., Astrophys. J. 666, 716 (2007) [arXiv:astro-ph/0701510].

2. J. Dunkley et al. [WMAP Collaboration], Observations: arXiv:0803.0586 [astro-ph], E. Komatsu et al. [WMAP Collaboration], arXiv:0803.0547 [astro-ph].

3. T. Padmanabhan, AIP Conf. Proc. 861, 179 (2006) [arXiv:astro-ph/0603114].

4. Roy Maartens, J. Phys.: Conf. Ser. 68, 012046 (2007).

5. R. Durrer and R. Maartens, Gen. Rel. Grav. 40, 301 (2008) [arXiv:0711.0077 [astro-ph]].

6. L. Fernández-Jambrina, R. Lazkoz, Phys. Rev. D 70121503 (2004) [arXiv:gr-qc/0410124]

7. F.J. Tipler, Phys. Lett. A 64, 8 (1977).

8. A. Królak, Class. Quant. Grav. 3, 267 (1986).

9. C.J.S. Clarke and A. Królak, Journ. Geom. Phys. 2, 127 (1985).

10. L. Fernández-Jambrina, R. Lazkoz, (accepted in PLB)

11. L. Fernández-Jambrina, R. Lazkoz, Phys. Rev. D 74, 064030 (2006) [arXiv:gr-qc/0607073]

12. M. P. Brown, and K. Austin, The New Physique, Publisher Name, Publisher City, 2000, pp. $212-213$.

13. M. P. Brown, and K. Austin, Appl. Phys. Letters 85, 2503-2504 (2000).

TABLE 2. Average turnover per shop: by type of retail organisation

\begin{tabular}{lrrrr}
\hline & $\begin{array}{c}\text { Single } \\
\text { outlet }\end{array}$ & $\begin{array}{c}\text { Small* } \\
\text { multiple }\end{array}$ & $\begin{array}{c}\text { Large } \\
\text { multiple }\end{array}$ & Total \\
\hline 1982 & 98 & 129 & 620 & 847 \\
1987 & 138 & 176 & 1000 & 1314 \\
1991 & 173 & 248 & 1230 & 1651 \\
$1998^{\dagger}$ & 200 & 300 & 1500 & 2000 \\
\hline
\end{tabular}

\footnotetext{
* 2-9 retail outlets
}

$\dagger$ predicted 
14. R. Wang, "Title of Chapter," in Classic Physiques, edited by R. B. Hamil, Publisher Name, Publisher City, 2000, pp. 212-213.

15. C. D. Smith and E. F. Jones, "Load-Cycling in Cubic Press," in Shock Compression of Condensed Matter-1999, edited by M. D. F. et al., AIP Conference Proceedings 505, American Institute of Physics, New York, 1999, pp. 651-654. 